\title{
SYNTHESIS, CRYSTAL STUDIES AND PHARMACOLOGICAL ROLE PREDICTION OF 3-IOD0-2- METHYL-1 PHENYL SULFONYL-1H INDOLE
}

\author{
RAMATHILAGAM CHINNATHAMBI ${ }^{1 *}$, VIJAYASRI SANTHANAM ${ }^{2}$, MANIVANNAN VADIVELU ${ }^{3}$, \\ UMARANI PADAGACHERI RAMACHANDRAN ${ }^{4}$, WAHEETA HOPPER ${ }^{2}$
}

${ }^{1}$ Department of Physics, D. G. Vaishnav College, Chennai, Tamil Nadu, India. ${ }^{2}$ Department of Biotechnology, Faculty of Engineering and Technology, SRM University, Kattankulathur, Chennai, Tamil Nadu, India. ${ }^{3}$ Department of Physics, Presidency College (Autonomous), Chennai, Tamil Nadu, India. ${ }^{4}$ Joint Director of Collegiate Education (Planning \& Development) (Retd) Department of Higher Education, Chennai, India. Email: cramathilagam1@gmail.com

Objective: Indole-based compounds have established many pharmacological applications. Indole acts as a starting compound for various medicinal preparations. Our objective is to determine the possible pharmacological roles of our crystallized indole-based ligand using computational approach. The activities studied are antibacterial, antitubercular, and antimelanoma.

Methods: The structure of the indole compound, CR2 was studied using single crystal X-ray diffraction technique. To predict the pharmacological activities, the structure-based docking method was followed. The protein targets were selected based on their important biological role in each activity mentioned above. The interactions between the targets and the crystal ligand were studied using a generic based docking algorithm. The significant pharmacophore features of the ligand were also reported.

Results: The ligand showed bonded and non-bonded interactions with the crucial amino acid residues of the active site of each target. The interactions and the binding energies were quite comparable to the targets' natural ligands.

Conclusion: We suggest through the computational approaches that the ligand may have antitubercular, antibacterial, and antimelanoma activities with respect to the targets considered. The new insights of this compound as predicted by the computational methods are believed to provide a platform for the futuristic pharmacological activities of this compound that can be further considered for wet lab techniques.

Keywords: Indole, Dihydrofolate reductase, DNA gyrase, Human B-Raf kinase, Docking, Pharmacophore, Single crystal X-ray diffraction.

(C) 2017 The Authors. Published by Innovare Academic Sciences Pvt Ltd. This is an open access article under the CC BY license (http://creativecommons. org/licenses/by/4. 0/) DOI: http://dx.doi.org/10.22159/ajpcr.2017.v10i3.16231

\section{INTRODUCTION}

Indole derivatives have displayed versatile pharmacological activities such as antimicrobial, antiviral, antihepatitis B virus, antifungal, antihuman immunodeficiency virus, antitubercular, antihypertensive, analgesic anti-inflammatory, anticancer, antidiabetic, and anti-convulsant agents [1]. These effects have been well proved in randomized controlled studies. Indole structure finds use in research as a starting material for the synthesis of larger, usually bioactive structures [2]. The importance of indole nucleus is well established in the field of pharmaceutical chemistry, plants, and animal biochemistry, and thus it has applications across a wide range of therapeutic areas [3]. Due to its pharmacological relevance, it is not surprising that the indole ring system has become an important building block or intermediate in the synthesis of many pharmaceutical agents. Since indole-based structures are capable of binding to many receptors with high affinity, they have been referred as privileged structures. Hence, the synthesis of several substituted indoles and the study of their crystal, molecular structure, and molecular docking studies continue to be an interesting field of research [4]. Docking is frequently used to predict the binding orientation of small molecules to the protein targets to predict the affinity and activity of the small molecule [5-7]. With this background information, a new indole derivative was synthesized. The synthesized compound was subjected to single crystal X-ray studies to investigate their molecular structure. The chemical structure of this derivative, 3-Iodo-2-methyl-1-phenylsulfonyl$1 \mathrm{H}$ indole (CR2) was mapped to possible anti-tubercular, antibacterial, and anti-melanoma activities using computational approaches. For this study, the three important biological targets such as dihydrofolate reductase (DHFR) of Mycobacterium tuberculosis, DNA gyrase B of Staphylococcus, and B-Raf kinase of human have been considered. The interactions of the ligand CR2 with the active site residues were studied using a generic algorithm based molecular docking, and the binding modes have been investigated. This was followed by the mapping of pharmacophore features of the ligand to understand the interactions in a better way. In addition, an in silico study of the ADME properties of the compound was conducted by investigating their Lipinski's parameters, topological polar surface area (TPSA), and percentage of absorption (\% ABS). Here, we report the synthesis, crystal studies and computational molecular docking studies of 3-Iodo-2-methyl-1-phenylsulfonyl-1H indole (CR2). The studies done through the computational methods will help in understanding the novel pharmacological roles of CR2. The study is also followed for the known inhibitors of these targets for comparison purpose.

\section{METHODS}

\section{Synthesis}

3-Iodo-2-methyl indole (5 $\mathrm{g}$ and $0.02 \mathrm{mmol}$ ) was dissolved in distilled benzene $(100 \mathrm{ml})$. To this, benzenesulfonyl chloride $(3.23 \mathrm{ml}$ and 0.025 $\mathrm{mmol}$ ) and $60 \%$ aqueous $\mathrm{NaOH}$ solution ( $40 \mathrm{~g}$ in $67.0 \mathrm{ml}$ ) were added along with tetra butyl ammonium hydrogen sulfate $(1.0 \mathrm{~g})$. This twophase system was stirred at room temperature for $2 \mathrm{hrs}$. It was then diluted with water $(200 \mathrm{ml})$ and the organic layer was separated. The aqueous layer was extracted with benzene $(2 \times 20 \mathrm{ml})$. The combined organic layer was dried $\left(\mathrm{Na}_{2} \mathrm{SO}_{4}\right)$. The benzene was then removed completely, and the crude product was recrystallized from methanol. 
X-ray crystallographic analysis

A single crystal of the title complex suitable for X-ray structural analysis is selected from the crystals obtained above. All measurements were made on a Bruker Kappa APEX-II diffractometer with graphite monochromated Mo-K $\alpha$ radiation of $0.71073 \AA$ Á. The structure was solved by direct methods and refined by full-matrix least squares on $\mathrm{F}^{2}$ using SHELXS97 [8]. Molecular graphics are drawn by PLATON [9]. All non-hydrogen atoms were refined anisotropically. The $\mathrm{H}$ atoms were introduced in calculated positions and refined with fixed geometry with respect to their carrier atoms [10]. The crystallographic data and refinement parameters are listed in Table 1 . Only the selected bond distances are given in Table 2 .

\section{Preparation of ligand structure}

The ligand structure was constructed using Chem BioDraw ultra 11.0 software (Molecular modeling and analysis; Cambridge Soft) shown in Fig. 1 and its Oak Ridge Thermal Ellipsoid Plot diagram is shown in Fig. 2.

\section{Computational study}

For the compound (CR2), the important physicochemical property which includes Lipinski's parameters, TPSA, and \% ABS were determined using molinspiration. To be pharmacologically favorable the compound should have pharmacokinetic and toxic properties. The rational drug designing approach relies on the prediction of molecular interactions between the target and the drug candidate. To propose the possible biological activities of CR2, the molecular docking was performed with the important targets of pharmacological importance using a generic algorithm based approach. The three main roles to be demonstrated using computational methods are antitubercular, antibacterial, and antimelanoma. The three-dimensional structures of the targets were downloaded from the PDB [11]. To elucidate the compound's antitubercular activity, the crystal structure of DHFR of M. tuberculosis in complex with NADPH and methotrexate (PDB code: 1DF7) was considered as target. The three-dimensional structure

Table 1: Crystal data of CR2 with important parameter information

\begin{tabular}{ll}
\hline Parameters & Data \\
\hline Molecular formula & $\mathrm{C}_{15} \mathrm{H}_{12} \mathrm{INO}_{2} \mathrm{~S}$ \\
MW & 397.22 \\
Crystal system & Monoclinic \\
Z & 4 \\
Space group & $\mathrm{P}_{1} / \mathrm{c}$ \\
Volume & $1435.49(6) \AA^{3}$ \\
Density & $1.838 \mathrm{Mg} \mathrm{m}^{-3}$ \\
Temp & $295 \mathrm{~K}$ \\
Unit cell dimensions & $\mathrm{a}=10.7068(3) \AA$ \\
& $\mathrm{b}=16.2670(4) \AA$ \\
& $\mathrm{c}=8.5147(2) \AA$ \\
Final R indices $(\mathrm{I}>2 \sigma[\mathrm{I}]) \mathrm{R} 1$ & 0.043 \\
Goodness-of-fit on F & 1.06 \\
Melting point & $395-397 \mathrm{~K}$ \\
\hline
\end{tabular}

MW: Molecular weight

Table 2: Selected bond distances of CR2

\begin{tabular}{llll}
\hline Bond & Distance $(\AA)$ & Bond & Distance $(\AA ̊)$ \\
\hline C1-C2 & $1.381(4)$ & C12-C13 & $1.387(5)$ \\
C2-C3 & $1.386(6)$ & C13-C14 & $1.392(4)$ \\
C3-C4 & $1.367(7)$ & C14-N1 & $1.411(3)$ \\
C4-C5 & $1.375(6)$ & C1-C6 & $1.380(4)$ \\
C5-C6 & $1.384(5)$ & C1-S1 & $1.759(3)$ \\
C7-C8 & $1.355(4)$ & C8-I1 & $2.050(3)$ \\
C8-C9 & $1.421(4)$ & C9-C14 & $1.399(4)$ \\
C9-C10 & $1.399(4)$ & N1-S1 & $1.667(2)$ \\
C10-C11 & $1.365(5)$ & O2-S1 & $1.416(3)$ \\
C11-C12 & $1.379(5)$ & O1-S1 & $1.411(3)$ \\
\hline
\end{tabular}

of DHFR of $M$. tuberculosis reveals opportunities for the design of novel tuberculosis drugs [12]. DHFR catalyzes the NADPH-dependent reduction of dihydrofolate to tetrahydrofolate, and this is essential for the synthesis of thymidylate, purines, and several amino acids. Inhibition of the enzyme's activity leads to the arrest of DNA synthesis and cell death. Hence, the inhibitors of this enzyme may control the population of this mycobacterium. The crystal structure of Staphylococcus Gyrase in complex with novobiocin (PDB code: 4URO) was used in docking studies to describe the antibacterial role [13]. DNA gyrase of bacteria is an essential topoisomerase that supercoils DNA through a process of strand breakage/resealing and DNA wrapping [14]. DNA gyrase is also the target for quinolone-based antibacterial agents which act by subverting the enzyme into a DNA damaging agent. Novobiocin binds to DNA gyrase, and blocks adenosine triphosphatase activity. The anti-melanoma activity was predicted using the interaction data between CR2 and Human B-Raf Kinase (PDB code: 3C4C), which is cocrystallized with an inhibitor named PLX4720 [15]. The binding modes of the compound with the protein targets were studied using a wellknown generic algorithm based iGemDock v2. 1. The pharmacological interactions between the compound and the targets were studied for the better understanding of ligand binding mechanisms. iGemDock is an interactive graphical generic evolutionary method for molecular docking for computing ligand conformations and orientations relative to the active site of target proteins [16]. The binding sites for the three targets were prepared by considering a radius of $8 \AA$ with co-crystal ligands as the center. The co-crystal ligands were also included in the docking procedure to check the efficacy of the protocol followed. All ligands were prepared for the correctness of their structures

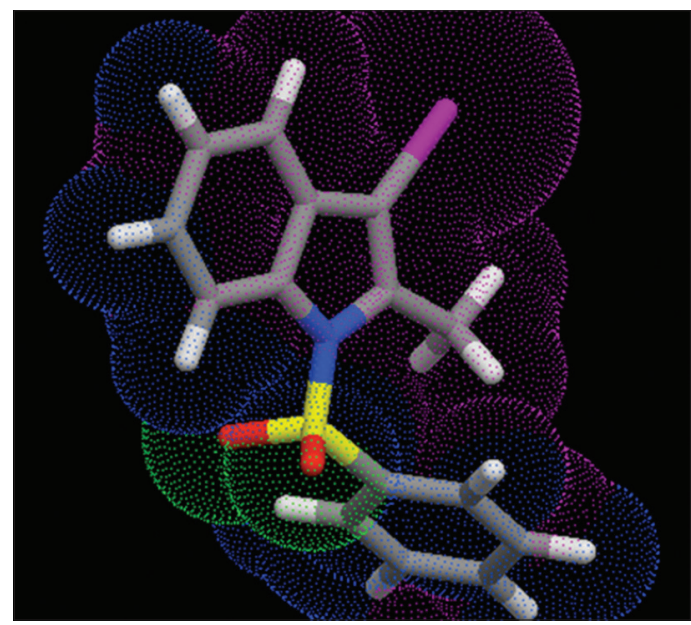

Fig. 1: Schematic diagram of CR2

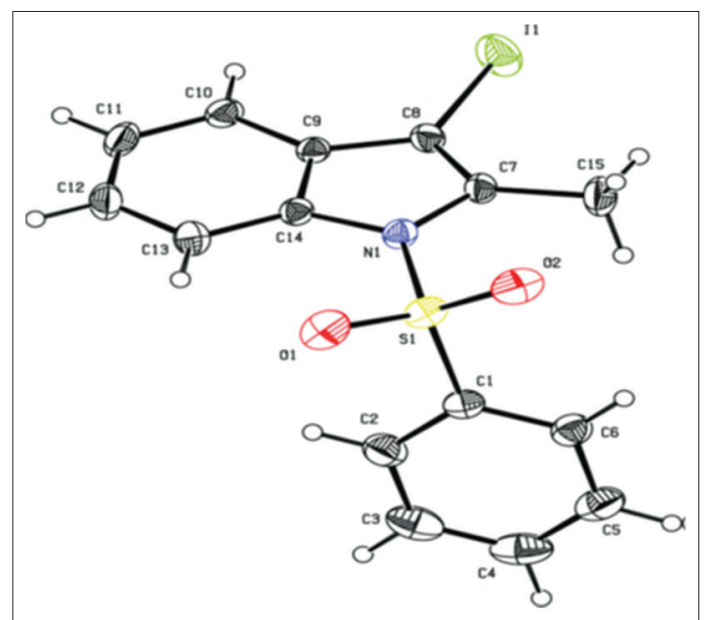

Fig. 2: Oak Ridge Thermal Ellipsoid Plot diagram of CR2 
beforedocking. Standard docking procedure with default genetic algorithm setting of 200 population size, 70 generations, and two solutions were followed. Each ligand was associated with a fitness score, which is the summation of bonded and non-bonded interactions. The non-bonded interactions include van der Waals and electrostatic contacts. The interaction profiles were created for each docking run, and the fitness scores were evaluated. The interactions were viewed using LigPlot ${ }^{+}$[17]. The molecular interactions can be better explained in terms of the features present in the ligand. This is technically called as pharmacophore and is defined as the spatial arrangement of the features that is essential for a molecule to interact with a specific target receptor. It is an important strategy followed in rational drug designing. The ligand-based pharmacophore model was created for CR2 and a comparison with the other ligands used in the docking study was also attempted to explain the interaction in a more contented manner. The pharmacophore study was done using multiple flexible alignments using PharmaGist [18].

\section{RESULTS AND DISCUSSION}

The oral ABS of the newly synthesized derivative was tentatively assessed using theoretical calculations following Lipinski's rule of five, which establishes that the ABS or permeation of an orally administered compound is more likely to be good if the drug satisfies the following criteria: (a) Hydrogen bond donors $\leq 5(\mathrm{OH}$ and $\mathrm{NH}$ groups); (b) hydrogen bond acceptors $\leq 10$ ( $\mathrm{N}$ and $\mathrm{O}$ atoms); (c) molecular weight $<500$; (d) calculated $\log \mathrm{P}<5$; (e) solubility (LogS) $>-4$. Compounds violating more than one of these rules may present bioavailability problems. The percentage of ABS was estimated using the equation: $\%$ ABS $=109-0.345 \times$ TPSA. The important physicochemical parameters are listed in Table 3. The prominent physicochemical properties of CR2 favor the Lipinski's rule of five, thus, proving its better oral ABS using molinspiration. It can also be noticed that the compound has satisfied the criteria specified in ADMET/Tox screening as tabulated in Table 4 proving that it is pharmacokinetically acceptable.

The docking results indicate that the compound has favorable bonded and non-bonded interactions with three targets specified. The fitness scores of the ligand with the three targets were comparable with that of co-crystal ligands (Table 5).

With respect to docking with DHFR, the common interactions observed between compound and co-crystal ligand were with D27,
Q28, F31, and nucleotide 5'-diphosphate (NDP). The contribution through van der Waals interaction superseded the bonded interaction. The co-crystal ligand methotrexate showed a better interaction which is evident from the fitness score. This proves that the docking protocol followed is appropriate for the given target. The ligand also exhibits a similar kind of interaction indicating its activity as an inhibitor of DHFR of M. tuberculosis. Methotrexate showed bonded and nonbonded interactions with the co-factor NDP. This interaction with cofactor could be a possible reason for its inhibition activity. Similarly, the compound CR2 also exhibited hydrogen bonding and van der Waals interaction with the co-factor. The carbon atoms present in the indole ring were found to have van der Waals interaction with the NDP. Similarly, iodine atom in the compound made an external linking with the NDP. Structurally, the ring carbon atoms of the ligands are exposed to the solvent surface. The ligand was found to have favorable hydrophobic interactions with I20, L24, Q28, R23, and F31. There was a hydrogen bond formation between I atom attached to the indole ring with $\mathrm{N}$ atom of A7. A hydrogen bond was also seen between $\mathrm{N}$ of the indole ring with the OD2 atom of D27, which is one of the most crucial active site residues with which the co-crystal ligand also shares interaction. Similarly, the 01 atom attached to $S$ had a bonded interaction with NE1 atom of W22. All these, amino acids were found to be in a binding pocket that was also preferred by the co-crystal inhibitor methotrexate. Through LigPlot, it was found that the hydrophobic interactions between NDP, P51, Q28, F31, D27, and I94 with these three ligands were found to be equivalent. The side chain interactions of the ligands with D27and I94 were found to be equivalent. The sulfur present in the ligand maintained van der Waals interaction with the NDP and D27. Most of the non-bonded interactions were contributed by the ring carbon atoms. Docking results indicate that the compound has strong interactions with the NDP and crucial amino acids present in the active site (Fig. 3). These results suggest that the CR2 could function as antitubercular agent targeting this protein.

To study the antibacterial effect, CR2 was docked with the crystal structure of DNA Gyrase B of Staphylococcus. The interactions were compared with the co-crystal ligand novobiocin, an antibiotic derived from Streptomyces knives. Hydrogen bonding with R200 (D chain) and van der Waals interactions with P87 and R144 were found to be a common interaction pattern between CR2 and novobiocin. Non-bonded interactions through van der Waals forces dominate the binding

Table 3: Physico-chemical properties of CR2

\begin{tabular}{|c|c|c|c|c|c|c|c|c|}
\hline Compound code & $\%$ ABS & $\operatorname{TPSA}^{\mathrm{a}}\left(\mathrm{A}^{2}\right)$ & nHBA $^{\text {a }}$ (NO) & nHBD $^{\mathrm{a}}(\mathrm{OHNH})$ & $\log \mathrm{P}^{\mathrm{a}}$ & MW $^{\mathrm{a}}$ & $\mathrm{n}$ violations & $\log S^{b}$ \\
\hline CR2 & 95.51 & 39.076 & 3 & 0 & 4.368 & 397.237 & 0 & -3.03 \\
\hline
\end{tabular}

Prediction servers: ${ }^{a}$ www.molinspiration.com; ${ }^{b}$ www. organic-chemistry.org/prog/peo. ABS: Absorption, TPSA: Topological polar surface area, MW: Molecular weight

Table 4: ADME/Tox screening for CR2

\begin{tabular}{|c|c|c|c|c|c|c|c|c|c|c|c|c|c|c|}
\hline Parameters & MW & Dr & $\mathrm{Ar}$ & FB & RB & $\# \mathbf{R}$ & RL & C & $\mathrm{nC}$ & $\mathrm{nC} / \mathrm{C}$ & \#Chrg & Chrg & $\log P$ & PSA \\
\hline Parameter standards & $200-400$ & $0-6$ & $0-12$ & $0-15$ & $0-50$ & $0-7$ & $0-12$ & $5-2$ & $>2$ & $0.1-1.0$ & $0-3$ & $(-2)-2$ & $(-2)-6$ & $0-150$ \\
\hline CR2 & 397.23 & 0 & 3 & 2 & 18 & 2 & 9 & 15 & 5 & 0.33 & 0 & 0 & 4.28 & 47.45 \\
\hline
\end{tabular}

MW: Molecular weight; Dr: Hydrogen donors; Ar: Hydrogen acceptors; FB: Flexible bonds; RB: Rigid bonds; \#R: Ring number; RL: Ring size; C: Atom number; Nc: Non carbons; NC/C: Ratio hetero/carbon; \#Chrg: Charge number; Chrg: Total charge; PSA: Polar surface area

Table 5: Docking scores (fitness energy kcal/mol) of ligands with the targets

\begin{tabular}{llllll}
\hline Target & Compound & Energy & VDW & HBond & Elec \\
\hline Dihydrofolate reductase of Mycobacterium tuberculosis 1DF7 & CR2 & -83.0247 & -65.0459 & -17.9788 & 0 \\
Gyrase of Staphylococcus 4URO & Methotrexate & -176.665 & -123.335 & -48.611 & -4.71911 \\
& CR2 & -94.4852 & -83.1669 & -11.3183 & 0 \\
Human B-Raf kinase 3C4C & Novobiocin & -148.471 & -116.699 & -31.7716 & 0 \\
& CR2 & -90.8108 & -81.2444 & -9.56638 & 0 \\
& PLX4720 & -111.463 & -105.208 & -6.25468 & 0 \\
\hline
\end{tabular}

Energy: Total energy, VDW: van der Waals, HBond: Hydrogen bond, Elec: Electrostatic forces 
between the ligands and the target, which amount to about $90 \%$ in case of CR2. The contribution of hydrogen bond to the total binding score is higher in case of novobiocin which could possibly be due to the presence of more oxygen atoms in its structure. The carbon atoms present in the indole ring of the compound are exposed to the solvent surface. The oxygen atoms attached to the sulfur atom of the compound is found to have hydrogen bond with side chains of D89 and Q91. These interactions are confined to chain A. Another noted interaction was also seen in the docking result which is the hydrogen bond with R200 of chain D (Fig. 4). Although the pattern of binding with the target varies between the ligands, the amino acids remain the same. That is, both the ligands bind to the crucial active site residues. The iodine atom present in the compound was not involved in any kind of interaction. The docking studies indicate the preference of ligands toward the same binding pocket. These observations recommend the candidate ligand CR2 to be antibacterial agent inhibiting this target protein.

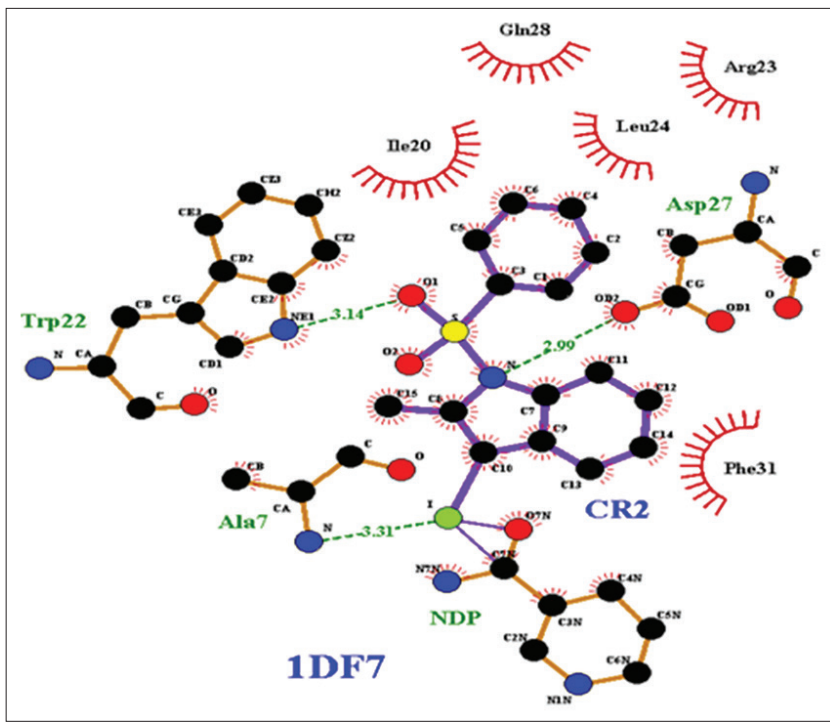

Fig. 3: Docking of CR2 with dihydrofolate reductase of Mycobacterium tuberculosis (Green dash lines-hydrogen bonds, red arcs-non-bonded van der Waals interaction)

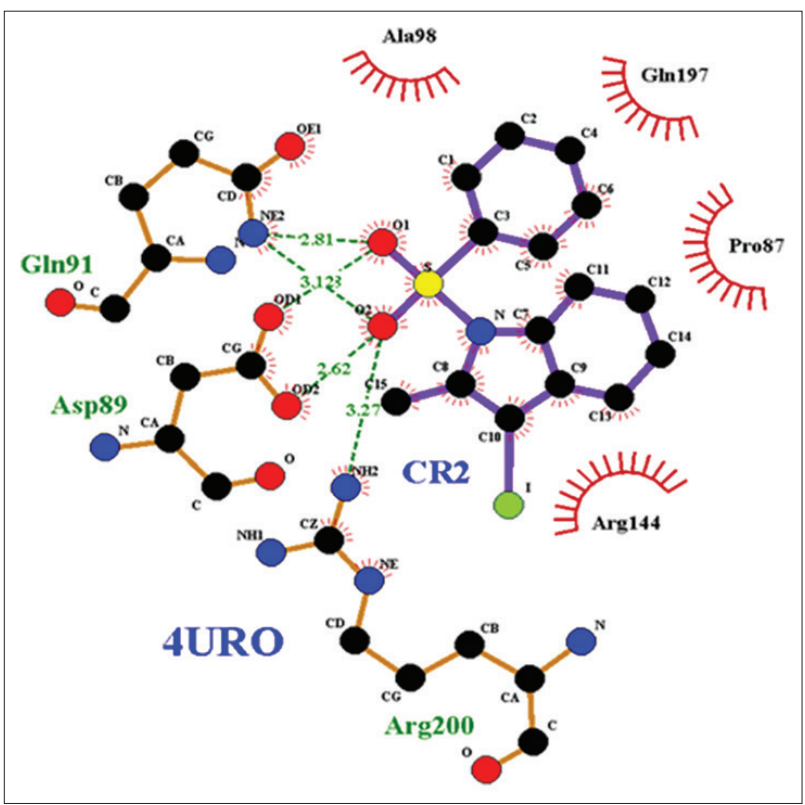

Fig. 4: Docking of CR2 with Gyrase of Staphylococcus showing interactions with crucial active site residues
The ligand, CR2 was docked with human Braf-kinase along with its selective inhibitor PLX4720. The docking results showed that the compound and co-crystal preferred the same binding site, which was spanned by L514, I527, K483, V471, T529, V471, and F583 (Fig. 5). Only the type of bonding differed between the ligands considered; the amino acids remain the same. The atom $\mathrm{O}_{2}$ of the ligand formed hydrogen bonds with NZ of K483 and N of D594. The docking results showed that the van der Waals interaction favored the binding of compound with protein. The iodine atom present in the compound was not involved in any kind of interaction. Both the ligands such as CR2 and selective inhibitor PLX4720 share some structural features in common such as indole ring, sulfonyl, and halogen groups. Based on the interaction with crucial residues in the active site comparable to the selective inhibitor, CR2 can be concluded to be an anti-melanoma through the inhibition of Braf-kinase. Overall docking studies showed that the van der Waals forces were the dominant key players favoring the interactions. Docking scores (fitness energy kcal/mol) of ligands with the targets is shown in Table 5. The pharmacophore model of CR2 shows seven spatial features: Three aromatic, two acceptors, one hydrophobic, and one positive group (Fig. 6). It can be noticed that the two aromatic features of CR2 contribute much to the non-bonded van der Waals interactions, which

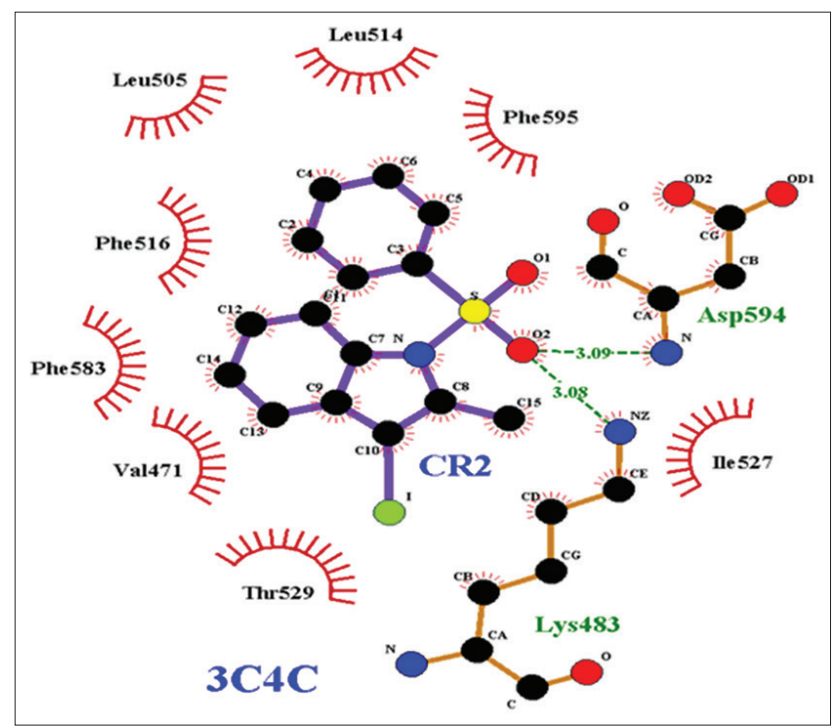

Fig. 5: Docking of CR2 with human Braf kinase showing bonded and non-bonded interactions with crucial active site residues

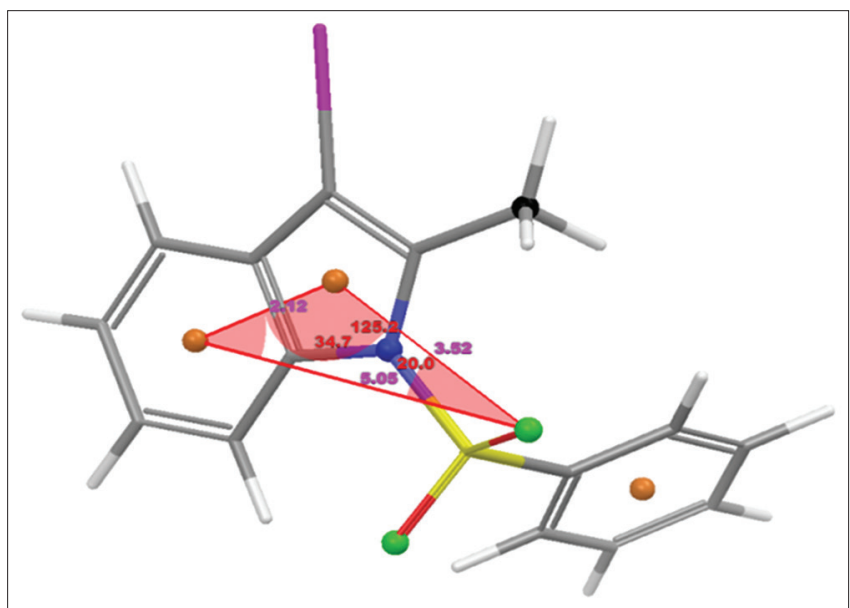

Fig. 6: Pharmacophore model of CR2 (orange-aromatic, greenacceptor, blue-positive, black-hydrophobic). The distance and angle measurements are shown only for the shared features 
were the dominant player of target interactions. The pharmacophoric features of all four ligands are tabulated (Table 6).

A pair-wise alignment was done between pharmacophore of CR2 and other co-crystal ligands to study the common pharmacophoric features shared by them. The score of the pair-wise alignment reflects their matched features. This pharmacophore information will guide us in designing lead compounds against the particular target. The shared pharmacophoric features between CR2 and the co-crystal ligands serve as a valid proof for a similar kind of interaction with the target proteins. The docking results also show the participation of the acceptor feature of CR2 in the hydrogen bond formation and the aromatic features in the non-bonded interactions with the active site residues thus validating the pharmacophore finding and the docking results. The superposition of pharmacophore models of all four ligands was also done to find the common features shared by the different structures (Fig. 7). The scores indicate that the features required by the co-crystal ligands to elicit the necessary biological activity against the specified targets are also present in the ligand CR2 thus supporting the docking protocol followed. All ligands share two aromatic features and an acceptor feature in common (Table 7).

The computational studies revealed that the ligand (CR2) has favorable bonded and non-bonded interactions with the crucial active site residues as comparable to the co-crystal inhibitors. They have similar kind of pharmacophoric features as like the known inhibitors.

Table 6: Pharmacophoric features of the ligands

\begin{tabular}{llllllllll}
\hline Molecule & Atoms & F & S & R & H & D & A & N & P \\
\hline CR2 & 32 & 7 & 7 & 3 & 1 & 0 & 2 & 0 & 1 \\
Novobiocin & 80 & 31 & 28 & 3 & 12 & 5 & 11 & 0 & 0 \\
PLX4720 & 41 & 12 & 11 & 3 & 2 & 2 & 4 & 0 & 1 \\
Methotrexate & 54 & 18 & 18 & 3 & 1 & 3 & 9 & 2 & 0 \\
\hline
\end{tabular}

F: Features, S: Spatial features, R: Aromatic, H: Hydrophobic, D: Donors,

A: Acceptors, N: Negatives, P: Positives

Table 7: Best pair-wise alignment between CR2 and other co-crystal ligands

\begin{tabular}{llllllllll}
\hline Molecules & F & S & R & H & D & A & N & P & Score \\
\hline CR2 and novobiocin & 4 & 4 & 3 & 0 & 0 & 1 & 0 & 0 & 10.5275 \\
CR2 and PLX4720 & 4 & 4 & 3 & 0 & 0 & 1 & 0 & 0 & 10.5217 \\
CR2 and methotrexate & 3 & 3 & 2 & 0 & 0 & 1 & 0 & 0 & 7.51522 \\
\hline
\end{tabular}

F: Features, S: Spatial features, R: Aromatic, H: Hydrophobic, D: Donors, A: Acceptors, N: Negatives, P: Positives

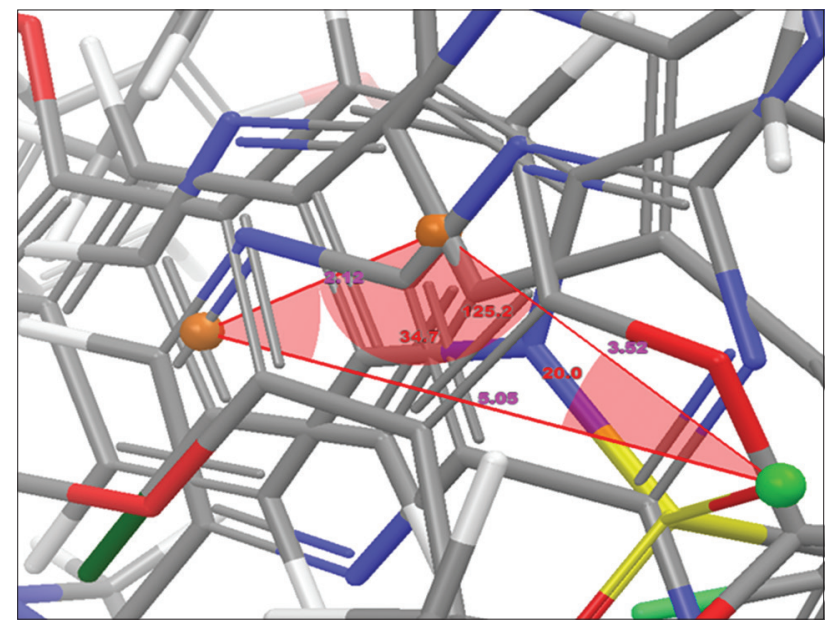

Fig. 7: Superposition of pharmacophoric models of all four ligands showing two aromatic and one acceptor features in common
Therefore, this compound will perform the function and the activity of the known and the co-crystal inhibitors with which it was compared. The various biological capabilities of other indole based compounds have been reported recently [19-21]. Hence, this work has extended the scope of developing the phenyl sulfonyl indole derivative compound as promising antitubercular, antibacterial, and anti-melanoma agents targeting the above-mentioned receptors. This compound could further be evaluated for these biological activities based on the supporting evidence from computational approaches.

\section{CONCLUSIONS}

The chemical structure of the synthesized compound was determined by single X-ray diffraction studies. The computer based methods are routinely and extensively used to reduce cost and time of drug discovery. Hence to identify the potential pharmacological role of the ligand CR2, the generic algorithm based molecular docking was performed. The studies of CR2 into the active site of the potential targets showed more favorable interactions. The van der Waals forces play the predominant role in the interactions. These facts are supported by the pharmacophore information of the compound. The pharmacophore details of the compound, CR2 indicate clearly that the pharmacological role is due to the spatial arrangement of two aromatic groups and an acceptor in a preferred geometry. The oral bioavailability is inversely proportional to TPSA. The predicted topological surface area of the compound supports the fact that it might have better oral ABS. The compounds with indole moiety have already been proved to have many biological roles. The interactions of CR2 with the crucial residues of the targets describe the potential applications of this compound as a promising antitubercular, antibacterial, and anti-melanoma candidate. The new insights of this compound as predicted by the computational methods are believed to provide a platform for the futuristic pharmacological activities of this compound that can be further considered for other experimental techniques.

\section{Supplementary information}

Supplementary data and figures of the compound are available from the IUCr electronic archives (Reference: GK2338).

\section{ACKNOWLEDGMENTS}

CR, SV, and WH thank SRM University for providing infrastructure to perform computational studies.

\section{REFERENCES}

1. Sharma V, Kumar P, Pathak DJ. Biological importance of the indole nucleus in recent years. A comprehensive review. J Heterocycl Chem 2010;47:491-502.

2. Kaushik NK, Kaushik N, Attri P, Kumar N, Kim CH, Verma AK, et al. Biomedical importance of indoles. Molecules 2013;18(6):6620-62.

3. Dhani R, Avinash A, Salenaagina SK, Teja MS, Masthanaiah P, Rathnam PR, et al. Indole: The molecule of diverse pharmacological activities. J Chem Pharm Res 2011;3(5):519-23.

4. Saravanan B, Saravanan RR, Manivannan V. Synthesis and molecular docking studies of indole based compound (2-methyl-1-phenylsulfonyl1h-Indol-3-1)phenylmethyl acetate to nicotinic acetylcholine receptors. J Chem Pharm Res 2012;4(6):3057-62.

5. Satheeshkumar C, Ravivarma C, Arjun P, Silambarasan V, Raaman N, Velmurugan D, et al. Synthesis, anti-microbial activity and molecular docking studies on triazolylcoumarin derivatives. J Chem Sci 2015;127(3):565-74

6. Swamy PV, Kambhampati PC, Chandrasekhar KB, Thirupathi G, Sujitha P, Kumar CG, et al. Synthesis, biological evaluation and molecular docking studies of some novel cyclopropane carbohydrazide derivatives as potential anticancer agents. J Chem Sci 2016;128(6):929-39.

7. Bathini R, Sivan SK, Fatima S, Manga V. Molecular docking, MM/ GBSA and 3D-QSAR studies on EGFR inhibitors. J Chem Sci 2016;128(7):1163-73.

8. Sheldrick GM. A short history of SHELX. Acta Crystallogr A 2008;64(1):112-22.

9. Spek AL. Structure validation in chemical crystallography. Acta Crystallogr D Biol Crystallogr 2009;65(2):148-55. 
10. Ramathilagam C, Saravanan V, Mohanakrishnan AK, Chakkaravarthi G, Umarani PR, Manivannan V. 3-Iodo-2-methyl-1phenylsulfonyl-1-hindole. Acta Crystallogr Sect E Struct Rep Online 2011;:E67:0632-42.

11. Berman HM, Westbrook J, Feng Z, Gilliland G, Bhat TN, Weissig H, et al. The Protein Data Bank. Nucleic Acids Res 2000;28(1):235-42.

12. Li R, Sirawaraporn R, Chitnumsub P, Sirawaraporn W, Wooden J, Athappilly $\mathrm{F}$, et al. Three-dimensional structure of $M$. tuberculosis dihydrofolate reductase reveals opportunities for the design of novel tuberculosis drugs. J Mol Biol 2000;295(2):307-23.

13. Lu J, Patel S, Sharma N, Soisson SM, Kishii R, Takei M, et al. Structures of kibdelomycin bound to Staphylococcus aureus GyrB and ParE showed a novel U-shaped binding mode. ACS Chem Biol 2014;9:2023-31.

14. Papillon J, Ménétret JF, Batisse C, Hélye R, Schultz P, Potier N, et al. Structural insight into negative DNA supercoiling by DNA gyrase, a bacterial type 2A DNA topoisomerase. Nucleic Acids Res 2013;41(16):7815-27.

15. Tsai J, Lee JT, Wang W, Zhang J, Cho H, Mamo S, et al. Discovery of a selective inhibitor of oncogenic B-Raf kinase with potent antimelanoma activity. Proc Natl Acad Sci U S A 2008;105(8):3041-6.

16. Hsu KC, Chen YF, Lin SR, Yang JM. iGEMDOCK: A graphical environment of enhancing GEMDOCK using pharmacological interactions and post-screening analysis. BMC Bioinformatics 2011;12 Suppl 1:S33.

17. Laskowski RA, Swindells MB. LigPlot: Multiple ligand-protein interaction diagrams for drug discovery. J Chem Inf Model 2011;51(10):2778-86.

18. Schneidman-Duhovny D, Dror O, Inbar Y, Nussinov R, Wolfson HJ. PharmaGist: A webserver for ligand-based pharmacophore detection. Nucleic Acids Res 2008;36:W223-8.

19. Sreenivasulu R, Sujitha P, Jadav SS, Ahsan MJ, Kumar CG, Raju RR. Synthesis, antitumor evaluation, and molecular docking studies of indole-indazolyl hydrazide-hydrazone derivatives. Monatsh Chem Chem Mon 2016;147:1-14.

20. Siddalingamurthy E, Mahadevan KM, Jagadeesh NM, Kumara MN. Synthesis and docking study of 3-(N-Alkyl/Aryl piperidyl) indoles with serotonin-5HT, H1 and CCR2 antagonist receptors. Int J Pharm Pharm Sci 2014;6(4):475-82.

21. Jagadeesh NM, Mahadevan KM, Kumara MN, Prashantha N. Synthesis and molecular docking study of N-alkyl/aryl-2-aryl indol-3yl glyoxylamides as novel anticancer agents. Int $\mathrm{J}$ Pharm Pharm Sci 2014;6(2):921-6 